\title{
Integrating nanoparticle quantification and statistical design of experiments for efficient HIV-1 virus-like particle production in High Five cells
}

\author{
Eduard Puente-Massaguer ${ }^{1}$ (D) $\cdot$ Martí Lecina $^{2} \cdot$ Francesc Gòdia $^{1}$ \\ Received: 17 October 2019 / Revised: 4 December 2019 / Accepted: 13 December 2019 / Published online: 6 January 2020 \\ (C) Springer-Verlag GmbH Germany, part of Springer Nature 2020
}

\begin{abstract}
The nature of enveloped virus-like particles (VLPs) has triggered high interest in their application to different research fields, including vaccine development. The baculovirus expression vector system (BEVS) has been used as an efficient platform for obtaining large amounts of these complex nanoparticles. To date, most of the studies dealing with VLP production by recombinant baculovirus infection utilize indirect detection or quantification techniques that hinder the appropriate characterization of the process and product. Here, we propose the application of cutting-edge quantification methodologies in combination with advanced statistical designs to exploit the full potential of the High Five/BEVS as a platform to produce HIV-1 Gag VLPs. The synergies between CCI, MOI, and TOH were studied using a response surface methodology approach on four different response functions: baculovirus infection, VLP production, VLP assembly, and VLP productivity. TOH and MOI proved to be the major influencing factors in contrast with previous reported data. Interestingly, a remarkable competition between Gag VLP production and non-assembled Gag was detected. Also, the use of nanoparticle tracking analysis and flow virometry revealed the existence of remarkable quantities of extracellular vesicles. The different responses of the study were combined to determine two global optimum conditions, one aiming to maximize the VLP titer (quantity) and the second aiming to find a compromise between VLP yield and the ratio of assembled VLPs (quality). This study provides a valuable approach to optimize VLP production and demonstrates that the High Five/BEVS can support mass production of Gag VLPs and potentially other complex nanoparticles.
\end{abstract}

Keywords High Five cells $\cdot$ Virus-like particle $\cdot$ Response surface methodology $\cdot$ Multiple-criteria decision analysis $\cdot$ Nanoparticle

\section{Introduction}

Virus-like particles (VLPs) have emerged as promising nanoparticles, particularly in the field of new recombinant vaccines (Mohsen et al. 2017). Compared with other conventional vaccines such as live-attenuated or inactivated viruses, VLPs can elicit potent antibody and $\mathrm{T}$ cell responses in a safe manner

Electronic supplementary material The online version of this article (https://doi.org/10.1007/s00253-019-10319-x) contains supplementary material, which is available to authorized users.

Eduard Puente-Massaguer eduard.puente@uab.cat

1 Departament d'Enginyeria Química, Biològica i Ambiental, Universitat Autònoma de Barcelona, Cerdanyola del Vallès, Barcelona, Spain

2 IQS School of Engineering, Universitat Ramón Llull, Barcelona, Spain since they do not contain genetic material from the virus itself (Andersson et al. 2018). Among the different proteins able to self-assemble as VLPs, the group-specific antigen (Gag) polyprotein has demonstrated a wide applicability in different research areas. These include their use as immunogens (Young and Ross 2006), drug delivery vehicles (Deo et al. 2015), scaffolds for surface antigen presentation (Vidigal et al. 2018), and research surrogates (Buonaguro et al. 2013). A common need in all these applications is the understanding of the production system itself, which is paramount to define the most adequate conditions for VLP production.

The baculovirus species Autographa californica multiple nucleopolyhedrovirus (AcMNPV) has been successfully used for the production of a variety of VLP types. The ease of handling, the high productivities associated and the capacity to accommodate large quantities of DNA have made the baculovirus expression vector system (BEVS) a very attractive platform (Fernandes et al. 2013; Gómez-Sebastián et al. 2014). From the different available insect cell lines, High Five 
cells have been reported to produce high VLP titers with a reduced level of contaminant baculovirus (Senger et al. 2009). In fact, Cervarix ${ }^{\circledR}$, the first VLP-based human papillomavirus vaccine approved, is produced using the High Five/BEVS.

Recent advances in the field of nanoparticle quantification and characterization have brought the opportunity to deepen into the understanding of the BEVS. In the frame of initiatives such as Process Analytical Technologies (PAT), the application of new and more sophisticated characterization technologies will be a powerful tool to increase the understanding of how the process conditions influence the quality of the final product. Nanoparticle tracking analysis (NTA) and flow virometry are gaining interest as tools to monitor the production of different particles, including VLPs (Nika et al. 2019; Pereira Aguilar et al. 2019). To date, most of the studies dealing with VLP production are performed with indirect quantification methods based on monomer detection, which hinder the appropriate assessment of the final product and the yields obtained (Haynes et al. 2009; Pillay et al. 2009; Visciano et al. 2011). In addition, the simultaneous production of extracellular vesicles (EV), which frequently fall in the size range of VLPs, is not often considered and may lead to an erroneous estimation of VLP titers. With the aim to deepen in the characterization of the different existing nanoparticle populations, the $e G F P$ was fused in frame to the gag gene from the human immunodeficiency virus serotype 1 (HIV-1).

Considering the information from previous reports, we decided to apply these novel techniques for optimizing the Gag VLP production process using the High Five/BEVS platform. Different methods are available for process improvement, from the classical one-variable-at-a-time approach to more sophisticated statistical design of experiments (DoE) (Puente-Massaguer et al. 2019). So far, VLP production studies have focused either on the former approach (Krammer et al. 2010; Pushko et al. 2017) or on simple factorial designs (Pillay et al. 2009; Pastor et al. 2019), limiting the analysis of higher order effects between the variables influencing VLP production. This is of special relevance since the use of lower or higher MOI than strictly required might reduce the maximum VLP yield obtained or be detrimental to the system, respectively. Here, we applied a response surface methodology (RSM) to evaluate the impact of cell concentration at infection (CCI), multiplicity of infection (MOI), and time of harvest $(\mathrm{TOH})$ on VLP production. To go a step further in process understanding, the inclusion of several responses during process optimization has shown successful results and applicability in different research areas (Pinzi et al. 2011; Honary et al. 2014; Bukzem et al. 2016). Particularly, the three responses considered in addition to VLP production were VLP assembly, baculovirus infection, and VLP productivity. These are of special relevance in processes with recombinant baculovirus since it is desirable to define production conditions encompassing high production yields with an acceptable ratio of correctly assembled Gag in the form of VLPs, but at the same time maintaining high productivities. A multiple-criteria decision analysis (MCDA) was implemented to combine the RSM-optimized responses into a global optimal set of conditions based on two criteria: quantity and quality. The two optimal production conditions were successfully validated, and the quantity optimum was compared with other Gag VLP production strategies using the High Five/BEVS. Finally, the correct formation and morphology of the VLPs produced was characterized using cryogenic transmission electron microscopy (cryo-TEM). The results presented in this work represent an advance with respect to current literature related to Gag VLP production in the High Five/BEVS and provide useful insights into nanoparticle-based process characterization.

\section{Materials and methods}

\section{Cell line and culture maintenance}

High Five (cat. num. B85502, Thermo Fisher Scientific, Grand Island, NY, USA) and Sf9 cells (cat. num. 71104, Merck, Darmstadt, Germany) were cultured in suspension in the lowhydrolysate content and animal origin-free Sf900III medium (Thermo Fisher Scientific). Both cell lines were routinely maintained at exponential growth phase in $125-\mathrm{mL}$ disposable polycarbonate Erlenmeyer flasks (Corning, Steuben, NY, USA) in $15 \mathrm{~mL}$ of medium and subcultured three times a week at a density of $2-4 \times 10^{5}$ cells $/ \mathrm{mL}$ for High Five cells (PuenteMassaguer et al. 2018) and $4-6 \times 10^{5}$ cells $/ \mathrm{mL}$ for Sf9 cells (Puente-Massaguer et al. 2020). All cultures were shaken at $130 \mathrm{rpm}$ using an orbital shaker (Stuart, Stone, UK) and maintained at $27^{\circ} \mathrm{C}$ in an incubator. Cell count and viability were performed using the Nucleocounter NC-3000 (Chemometec, Allerod, Denmark) according to manufacturer's instructions.

\section{Baculovirus stock preparation and titration}

The recombinant Autographa californica multicapsid nucleopolyhedrovirus (AcMNPV), containing the HIV-1 Gag matrix protein fused in frame to eGFP (BV-GageGFP) (Hermida-Matsumoto and Resh 2000) and under the control of the polyhedrin promoter, was constructed using the BaculoGold system (BD Biosciences, San Jose, CA, USA). Baculovirus amplification was performed in the $\mathrm{Sf} 9$ cell line (cat. num. 71104, Merck, Darmstadt, Germany) cultured in Sf900III medium by infection at $3 \times 10^{6}$ cells $/ \mathrm{mL}$, a multiplicity of infection (MOI) of 0.1 , and harvest at 96 hours post infection (hpi).

Titration of baculovirus infectious particles was conducted using the plaque assay method. Briefly, $2 \mathrm{~mL}$ of $\mathrm{Sf} 9$ cells at a 
concentration of $0.5 \times 10^{6} \mathrm{cell} / \mathrm{mL}$ was plated per well in 6well plates (Nunc, Roskilde, Denmark) and left to attach for 30 min at RT. Then, the Sf900III medium was aspired and Sf9 cells were infected with the addition of $1 \mathrm{~mL}$ per well of serial dilutions $\left(10^{-4}\right.$ to $\left.10^{-9}\right)$ of the BV-GageGFP samples. Six well plates were incubated at very mild mixing conditions during $1 \mathrm{~h}$ to homogeneously distribute the baculoviruses. Afterwards, baculovirus-containing supernatants were aspired and $2 \mathrm{~mL}$ of a type VII-A agarose (Merck) solution warmed at $42{ }^{\circ} \mathrm{C}$ was added per well to cover the infected cells. The agarose solution was prepared by mixing an ultrapure water solution containing 3\% w/v agarose with Sf900III medium supplemented with $10 \%$ fetal bovine serum (FBS) at a 1:1 ratio. After agarose cooling, $2 \mathrm{~mL}$ of Sf900III medium supplemented with $10 \%$ FBS was added per well and the plates were incubated for 5 days at $27{ }^{\circ} \mathrm{C}$ without agitation. The concentration of infectious baculovirus particles, expressed as plaque forming units (pfu), was measured by visual inspection after $3 \mathrm{~h}$ staining with a $0.3 \%$ w/v Neutral Red solution (Merck) prepared in DPBS.

\section{Cell growth and baculovirus infection conditions}

Cell growth and infection studies were performed in High Five cell cultures seeded at $3 \times 10^{5}$ cell $/ \mathrm{mL}$ in $15 \mathrm{~mL}$ and maintained at mid-exponential phase by cell passaging every $48 \mathrm{~h}$. Several MOI, cell concentrations at infection (CCI), and times of harvest $(\mathrm{TOH})$ were evaluated and the different parameter combinations were defined according to a DoE-based approach. Samples were taken every $12-24 \mathrm{~h}$ to monitor viable cell concentration, cell viability, baculovirus infection, intra- and extracellular GageGFP expression, and nanoparticle production. Samples were harvested by centrifugation at $3000 \times \mathrm{g}$ for $5 \mathrm{~min}$ and supernatants were maintained at $4{ }^{\circ} \mathrm{C}$ until analysis. Cell pellets were stored at $-20^{\circ} \mathrm{C}$.

\section{Flow cytometry}

The percentage of GageGFP expressing cells was assessed using a BD FACS Canto II flow cytometer equipped with a 488-nm laser configuration (BD Biosciences). Briefly, $0.3 \mathrm{~mL}$ of infected cell cultures was harvested by centrifugation at $300 \times g$ for $5 \mathrm{~min}$ and fixed using $4 \% p$-formaldehyde for $10 \mathrm{~min}$. Cells were then centrifuged at $500 \times \mathrm{g}$ for $5 \mathrm{~min}$ to remove $p$-formaldehyde, resuspended in $0.3-\mathrm{mL}$ ice-cold DPBS (Thermo Fisher Scientific), and maintained at $4{ }^{\circ} \mathrm{C}$ until further analysis.

\section{Fluorescence confocal microscopy}

BV-GageGFP-infected High Five cells were observed using a TCS SP5 confocal microscope (Leica, Wetzlar, Germany). Cells were stained with $0.05 \% \mathrm{v} / \mathrm{v}$ of CellMask ${ }^{\mathrm{TM}}$ and $0.1 \%$ v/v of Hoechst (Thermo Fisher Scientific) to visualize the lipid membrane and cell nucleus, respectively. A washing step prior to observation was performed by centrifugation at $300 \times g$ during $5 \mathrm{~min}$, and then cells were resuspended in fresh Sf900III medium. Samples were placed in 35-mm glass bottom Petri dishes with a 14-mm microwell (MatTek Corporation, Ashland, MA, USA) for visualization. The VLP production process was monitored during $6.5 \mathrm{~min}$ at $48 \mathrm{hpi}$ using the Resonant scanner mode of the confocal microscope (Leica).

\section{GageGFP quantification}

The amount of GageGFP produced by High Five infected cells was measured intra- and extracellularly by spectrofluorometry. Supernatants of GageGFP producing cells were harvested by centrifugation at $3000 \times g$ for $5 \mathrm{~min}$ and maintained at $4{ }^{\circ} \mathrm{C}$ until analysis. Intracellular GageGFP content was evaluated by disruption of pelleted cells by means of three freeze-thaw cycles $\left(2.5 \mathrm{~h}\right.$ frozen at $-20{ }^{\circ} \mathrm{C}$ and thawed at $37{ }^{\circ} \mathrm{C}$ for $0.5 \mathrm{~h}$ ). Samples were vortexed for $5 \mathrm{~s}$ between cycles. Lysed pellets were resuspended in $0.5 \mathrm{~mL}$ of TMS buffer (50-mM Tris-HCl, $150-\mathrm{mM} \mathrm{NaCl}, 2-\mathrm{mM} \mathrm{MgCl}_{2}$, $\mathrm{pH} 8.0$ ), centrifuged at $13,700 \times \mathrm{g}$ for $20 \mathrm{~min}$ and kept at $4{ }^{\circ} \mathrm{C}$. GageGFP fluorescence was measured with a Cary Eclipse fluorescence spectrophotometer (Agilent Technologies, Santa Clara, CA, USA) at RT as follows: $\lambda_{\mathrm{ex}}=488 \mathrm{~nm}(5-\mathrm{nm}$ slit $), \lambda_{\mathrm{em}}=500-530 \mathrm{~nm}$ (10-nm slit). Relative fluorescence unit values (RFU) were calculated by subtracting fluorescence unit values of BV-GageGFP-infected and non-infected cell samples. RFU values were converted into GageGFP VLPs as described by Gutiérrez-Granados and co-authors (Gutiérrez-Granados et al. 2013) and detailed in the following equation:

GageGFP VLP $($ particles $/ \mathrm{mL})=1.5310^{8} \times$ RFU

The Sf900III medium and a $0.1 \mathrm{mg} / \mathrm{mL}$ quinine sulfate solution were used as control patterns to normalize RFU values in samples between different experiments.

\section{VLP quantification using nanoparticle tracking analysis}

A NanoSight NS300 (Malvern Panalytical, Malvern, UK) was used to measure fluorescent and non-fluorescent particles by nanoparticle tracking analysis (NTA). Samples were diluted in $0.22-\mu \mathrm{m}$-filtered DPBS and continuously injected into the device chamber through a pump at an average concentration of $10^{8}$ particles $/ \mathrm{mL}$ (20-60 particles/frame). Videos of $60 \mathrm{~s}$ from independent triplicate measurements were analyzed with the NTA 3.2 software at RT. The nanoparticle number was evaluated in the 100-250-nm range. 


\section{Nanoparticle quantification using flow virometry}

Fluorescent and non-fluorescent nanoparticles were also analyzed using a CytoFlex LX (Beckman Coulter, Brea, CA, USA) equipped with a 488-nm blue laser for fluorescent particle detection and a $405 \mathrm{~nm}$ laser/violet side scatter configuration for improved nanoparticle resolution. Samples were diluted in 0.22- $\mu \mathrm{m}-$ filtered DPBS and triplicate measurements from independent samples were analyzed with the CytExpert 2.3 software at RT.

\section{Analytical ultracentrifugation}

Supernatants of BV-GageGFP-infected High Five cells harvested at the different conditions of the DoE were ultracentrifuged in a double cushion of $5 \mathrm{~mL}$ of $25 \%$ and $8 \mathrm{~mL}$ of $45 \%(\mathrm{w} / \mathrm{v})$ sucrose (Merck) solution prepared in DPBS and DMEM (Thermo Fisher Scientific), respectively. Five milliliters of supernatants from triplicate experiments were loaded in ultracentrifuge tubes (Beckman Coulter) and filled to the top with sterile DPBS. Sample ultracentrifugation was performed in a Beckman Optima L100XP centrifuge (Beckman Coulter) equipped with a SW-32Ti rotor at $31,000 \mathrm{rpm}$ for $2.5 \mathrm{~h}$ at $4{ }^{\circ} \mathrm{C}$. Samples were taken from each ultracentrifuge fraction, and pellets were resuspended in $100 \mu \mathrm{L}$ of DPBS and kept overnight at $4{ }^{\circ} \mathrm{C}$. All samples were stored at $-20^{\circ} \mathrm{C}$ until their measurement by spectrofluorometry and at $4{ }^{\circ} \mathrm{C}$ for nanoparticle analysis by flow virometry. The same procedure was applied to characterize the optimal conditions obtained with the DoE and the MCDA approach.

\section{Cryogenic transmission electron microscopy}

VLP morphology was assessed using a transmission electron microscope equipped for sample cryogenics (cryo-TEM). Briefly, $4 \mu \mathrm{L}$ of sample was blotted onto EMR Holey carbon films on 400-mesh copper grids (Micro to Nano, Wateringweg, Netherlands) previously subjected to a glow discharge treatment in a PELCO easiGlow ${ }^{\mathrm{TM}}$ Discharge Cleaning System (PELCO, Fresno, CA, USA). Deposited samples were then plunged into liquid ethane at $-180{ }^{\circ} \mathrm{C}$ using a Leica EM GP workstation (Leica) and observed in a JEM-2011 TEM operating at $200 \mathrm{kV}$ (Jeol Ltd., Akishima, Tokyo, Japan).

\section{Multiple optimization of VLP production conditions}

A response surface methodology (RSM) was applied to assess the effect of the main factors influencing the BEVS: CCI (cell/mL), MOI ( $\mathrm{pfu} / \mathrm{cell}$ ), and $\mathrm{TOH}(\mathrm{h})$ on different responses. The selected DoE responses were baculovirus infection (\% of infected cells), VLP production (fluorescent particle/mL), GageGFP polyprotein assembled in the form of VLPs (\% of GageGFP assembled as VLPs), and VLP productivity (fluorescent particle/mL h). VLP production and VLP productivity responses were transformed to $\log$ values using the logarithmic regression to reduce the funnel shape effect of the residuals. This transformation compensated the increasing differences in standard deviation associated to higher figures, a common issue when simultaneously considering values with more than 1000-fold difference (Barbur et al. 1994). In parallel, baculovirus infection and VLP assembly responses were converted using the logistic regression to limit the possible values of the functions to the $0-100 \%$ range:

$Y_{\mathrm{n}}=\log \left(\frac{y_{\mathrm{n}}}{100-y_{\mathrm{n}}}\right)$

where $Y_{\mathrm{n}}$ is the converted response and $y_{\mathrm{n}}$ is the experimental response in natural values.

A Box-Behnken design was used to determine the influence of $\mathrm{CCI}, \mathrm{MOI}$, and $\mathrm{TOH}$ on each evaluated response. These variables were screened at a low $(-1)$, medium $(0)$, and high level $(+1)$ as indicated in Table 1 . The different levels were linearly related for $\mathrm{CCI}$ and $\mathrm{TOH}$ and exponentially for MOI, always in an equidistant manner. The results obtained for each response were fitted to a second-order polynomial by the least squares method (Eq. 3):

$$
\begin{aligned}
Y= & \beta_{0}+\beta_{1} \cdot \mathrm{CCI}+\beta_{2} \cdot \mathrm{MOI}+\beta_{3} \cdot \mathrm{TOH}+\beta_{4} \cdot \mathrm{CCI} \\
& \cdot \mathrm{MOI}+\beta_{5} \cdot \mathrm{CCI} \cdot \mathrm{TOH}+\beta_{6} \cdot \mathrm{MOI} \cdot \mathrm{TOH}+\beta_{7} \\
& \cdot \mathrm{CCI}^{2}+\beta_{8} \cdot \mathrm{MOI}^{2}+\beta_{9} \cdot \mathrm{TOH}^{2}+\varepsilon
\end{aligned}
$$

where $Y$ is the response variable, $\beta_{0}$ is the model intercept term, $\beta_{1}-\beta_{9}$ are the model coefficients, and $\varepsilon$ is the experimental error. Fitting of the different equations based on Eq. 3 was performed with the $\mathrm{R}$ software ( $\mathrm{R}$ Foundation for Statistical Computing, Vienna, Austria). Three-dimensional plots were constructed to facilitate model interpretation.

The most common DoE approach involves the optimization of a single response. However, such analysis may not be enough for the BEVS, since the definition of a production condition cannot be accurately predicted from an individual property. Then, the objective of including more than one response in the study was to determine global conditions integrating different key aspects of the BEVS. To this purpose, a multiple-criteria decision analysis (MCDA) based on desirability functions was implemented. The different responses were transformed to a dimensionless desirability scale $\left(d_{n}\right)$ ranging between 0 and 1 :

$$
\begin{aligned}
& d_{n}=0 \text { if } y_{n}<\min _{n} \\
& d_{n}=\left(\frac{y_{n}-\min _{n}}{\max _{n}-\min _{n}}\right)^{w} \text { if } \min _{n} \leq y_{n} \leq \max _{n} \\
& d_{n}=1 \text { if } y_{n}>\max _{n}
\end{aligned}
$$


Table 1 Matrix design, independent variable levels, and regression coefficients for the Box-Behnken design. VLP concentration values were quantified using nanoparticle tracking analysis

\begin{tabular}{|c|c|c|c|c|c|c|c|c|}
\hline \multicolumn{9}{|c|}{ Box-Behnken design } \\
\hline \multirow[b]{2}{*}{ Experimental run } & \multicolumn{3}{|c|}{ Variables } & \multicolumn{4}{|c|}{ Responses } & \\
\hline & $\begin{array}{l}\text { CCI }\left(10^{6}\right. \\
\text { cell } / \mathrm{mL})\end{array}$ & $\begin{array}{c}\text { MOI } \\
\text { (pfu/cell) }\end{array}$ & $\mathrm{TOH}(\mathrm{h})$ & $\begin{array}{c}\text { Infection } \\
(\%)\end{array}$ & $\begin{array}{c}\text { VLP production } \\
\left(10^{8} \text { fluorescent }\right. \\
\text { particle } / \mathrm{mL})\end{array}$ & $\begin{array}{c}\text { VLP } \\
\text { assembly (\%) }\end{array}$ & $\begin{array}{l}\text { VLP productivity } \\
\left(10^{6} \text { fluorescent }\right. \\
\text { particle } / \mathrm{mL} \cdot \mathrm{h})\end{array}$ & \\
\hline 1 & 1.5 & 0.01 & 84 & 98.5 & 345.5 & 14.4 & 411.3 & \\
\hline 2 & 0.5 & 0.22 & 84 & 99.5 & 118.1 & 31.5 & 140.6 & \\
\hline 3 & 1.5 & 0.22 & 54 & 99.2 & 230.0 & 17.1 & 420.0 & \\
\hline 4 & 1.5 & 0.22 & 54 & 99.6 & 214.8 & 15.2 & 410.0 & \\
\hline 5 & 2.5 & 5 & 54 & 99.3 & 320.2 & 13.4 & 593.0 & \\
\hline 6 & 1.5 & 5 & 84 & 99.4 & 265.3 & 17.5 & 315.8 & \\
\hline 7 & 1.5 & 0.01 & 24 & 3.3 & 0.2 & 98.2 & 0.9 & \\
\hline 8 & 1.5 & 5 & 24 & 95.5 & 11.6 & 57.2 & 48.3 & \\
\hline 9 & 2.5 & 0.01 & 54 & 21.7 & 8.7 & 14.6 & 16.1 & \\
\hline 10 & 0.5 & 0.01 & 54 & 97.8 & 34.3 & 37.7 & 63.4 & \\
\hline 11 & 0.5 & 0.22 & 24 & 56.4 & 1.9 & 94.6 & 7.7 & \\
\hline 12 & 2.5 & 0.22 & 84 & 99.5 & 465.4 & 13.2 & 554.1 & \\
\hline 13 & 0.5 & 5 & 54 & 99.7 & 101.8 & 34.3 & 188.5 & \\
\hline 14 & 1.5 & 0.22 & 54 & 99.4 & 200.0 & 13.1 & 370.0 & \\
\hline 15 & 2.5 & 0.22 & 24 & 30.7 & 1.1 & 93.9 & 4.4 & \\
\hline \multicolumn{9}{|c|}{ Analysis } \\
\hline Function & \multicolumn{2}{|c|}{$F$ test, $p$-value } & $\begin{array}{l}\text { LOF test, } p \text { - } \\
\text { value }\end{array}$ & $R^{2}$ & Adjusted $R^{2}$ & Predicted $R^{2}$ & & \\
\hline Infection & \multicolumn{2}{|c|}{$<0.01$} & 0.1 & 95.0 & 90.0 & 68.7 & & \\
\hline VLP production & \multicolumn{2}{|c|}{$<0.01$} & 0.05 & 99.7 & 99.1 & 95.0 & & \\
\hline VLP assembly & \multicolumn{2}{|c|}{$<0.01$} & 0.06 & 94.9 & 92.1 & 79.9 & & \\
\hline VLP productivity & \multicolumn{2}{|c|}{$<0.01$} & 0.05 & 99.5 & 98.7 & 92.9 & & \\
\hline Model & \multicolumn{2}{|c|}{ Infection } & \multicolumn{2}{|c|}{ VLP production } & \multicolumn{2}{|c|}{ VLP assembly } & \multicolumn{2}{|c|}{ VLP productivity } \\
\hline Parameters & Coefficient & $p$-value & Coefficient & $p$-value & Coefficient & $p$-value & Coefficient & $p$-value \\
\hline Constant & 4.7 & $<0.01$ & 5.4 & $<0.01$ & -1.4 & $<0.01$ & 6.0 & $<0.01$ \\
\hline $\mathrm{CCI}$ & -0.9 & 0.03 & 0.1 & $>0.1$ & -0.5 & 0.04 & 0.1 & $>0.1$ \\
\hline $\mathrm{CCI}^{2}$ & $N S$ & $>0.1$ & -0.8 & $<0.01$ & $N S$ & $>0.1$ & -0.8 & $<0.01$ \\
\hline MOI & 2.0 & $<0.01$ & 1.1 & $<0.01$ & -0.5 & 0.04 & 1.1 & $<0.01$ \\
\hline $\mathrm{MOI}^{2}$ & -1.0 & 0.08 & -0.6 & $<0.01$ & $N S$ & $>0.1$ & -0.6 & $<0.01$ \\
\hline $\mathrm{TOH}$ & 2.6 & $<0.01$ & 2.3 & $<0.01$ & -2.0 & $<0.01$ & 2.0 & $<0.01$ \\
\hline $\mathrm{TOH}^{2}$ & -1.8 & $<0.01$ & -1.7 & $<0.01$ & -1.9 & $<0.01$ & -1.5 & $<0.01$ \\
\hline $\mathrm{CCI} \cdot \mathrm{MOI}$ & 1.1 & 0.06 & 0.6 & $<0.01$ & $N S$ & $>0.1$ & 0.6 & $<0.01$ \\
\hline $\mathrm{CCI} \cdot \mathrm{TOH}$ & $N S$ & $>0.1$ & 0.6 & $<0.01$ & $N S$ & $>0.1$ & 0.5 & $<0.01$ \\
\hline $\mathrm{MOI} \cdot \mathrm{TOH}$ & -1.4 & 0.02 & -1.1 & $<0.01$ & 1.0 & $<0.01$ & -1.1 & $<0.01$ \\
\hline
\end{tabular}

$N S$ non-statistically significant

where $y_{n}$ is the predicted response of the fitted equation, $d_{n}$ is the desirability response function, $w$ accounts for the weight value, and $\min _{n}$ and $\max _{n}$ are the minimum and maximum acceptable values of $y_{n}$, respectively. The weight ( $w$ value) 
depended on the relative importance assigned to each individual response. Then, all the different desirability response functions under study were combined into a single equation, denoted as overall desirability (OD):

$\mathrm{OD}=\sqrt[N]{\prod_{1}^{N} d_{n}}$

where OD is the overall desirability function to be maximized and $N$ is the number of responses studied in the optimization process.

\section{Statistical analyses}

The experimental design and statistical analyses of the different equations were performed using $\mathrm{R}$ software. The quality of the regression of the fitted equations was evaluated with the $R^{2}$ and adjusted $R^{2}$ coefficients in percentage units. An analysis of variance (ANOVA) $F$ test was used to determine the significance of the equations and the individual coefficients were assessed with a $t$ test. The lack-of-fit (LOF) test was used to evaluate differences between experimental and pure error of the fitted equations. In all analyses, $p$ values of 0.05 and 0.1 were considered statistically significant with $95 \%$ and $90 \%$ confidence, respectively.

\section{Results}

\section{Definition of the experimental space boundaries}

Initially, the growth of High Five cells was characterized to determine an adequate range of viable cell concentration at infection (CCI) for the DoE. Cells maintained a high viability (>90\%) until $96 \mathrm{~h}$ of culture, coinciding with the peak of viable cell concentration. In these conditions, cells reached a maximum of $7.3 \pm 0.2 \times 10^{6}$ cells $/ \mathrm{mL}$ and a doubling time $\left(T_{\mathrm{d}}\right)$ of $18.7 \pm 0.5 \mathrm{~h}$ (Fig. 1). Considering the extent of the exponential phase and the $T_{\mathrm{d}}$, the range for cell concentration at infection was restricted to $0.5-2.5 \times 10^{6}$ cells $/ \mathrm{mL}$. Some difficulties were encountered when defining the different levels of multiplicity of infection (MOI) and time of harvest (TOH) since very little information on the use of the BEVS with High Five cells in the Sf900III medium was available. The ranges for both variables were eventually fixed according to previous experience with this system (data not shown) and based on studies of the High Five/BEVS in other cell culture media (Sander and Harrysson 2007; Pillay et al. 2009). The MOI experimental range was fixed between 0.01 and 5 with the aim to screen low and high MOI conditions in the same DoE. In the case of $\mathrm{TOH}$, the boundaries were defined as 24-84 hpi. DoE space boundaries did not involve high CCI out of the exponential phase or medium exchange in order to

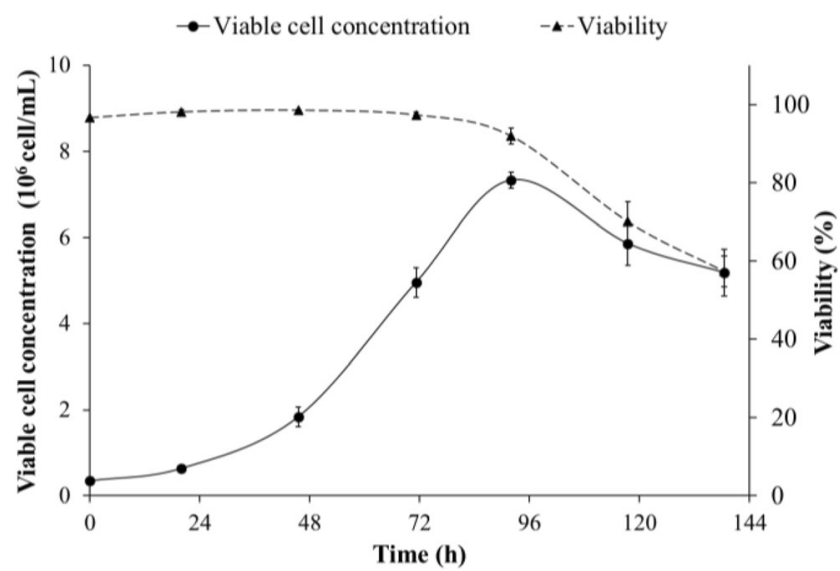

Fig. 1 Cell growth and viability profiles of High Five cells in Sf900III medium in batch culture. Exponentially growing cells were seeded at $0.3 \times 10^{6}$ cells $/ \mathrm{mL}$ in 125 -mL flasks. Mean values \pm standard deviation of triplicate experiments are represented

simplify their industrial application, but these conditions were evaluated as a contrast to the optimal conditions obtained as further presented.

\section{Combining RSM and MCDA to define the optimal VLP production conditions}

A three-variable (CCI, MOI, and TOH) Box-Behnken RSM was constructed and the following objective responses were analyzed: baculovirus infection efficiency, VLP production, VLP assembly, and VLP productivity. This experimental design was selected among different candidates due to its variance distribution and spatial properties (Cervera et al. 2015a; Gutiérrez-Granados et al. 2016; Puente-Massaguer et al. 2018). The resulting matrix consisted in 15 experiments including a triplicated center point to account for the inherent variability of the system (Table 1). The different datasets were adjusted to the second-order polynomial functions by the least squares method according to Eq. 3. The statistical significance of the different functions and their associated coefficients was confirmed by ANOVA (Table 1). Each function was used to construct three-dimensional plots for a better comprehension of the synergies between CCI, MOI, and TOH (Fig. 2). As expected, baculovirus infection progressed more rapidly with increasing MOI (Fig. 2a-c). However, the pace of infection decreased when using the same MOI at higher CCI. A similar behavior was observed between VLP production and VLP productivity (Fig. 2d-i). Low MOI required longer TOH to achieve higher VLP yields, whereas higher MOI shortened this period. In both cases, the best production and productivity titers were obtained in the $1.5-2.5 \times 10^{6}$ cell $/ \mathrm{mL}$ CCI range. $\mathrm{TOH}$ also proved to be the most significant variable regarding the amount of GageGFP monomer assembled in the form of VLPs (Fig. 2j-1). Increasing TOH and MOI substantially 
a
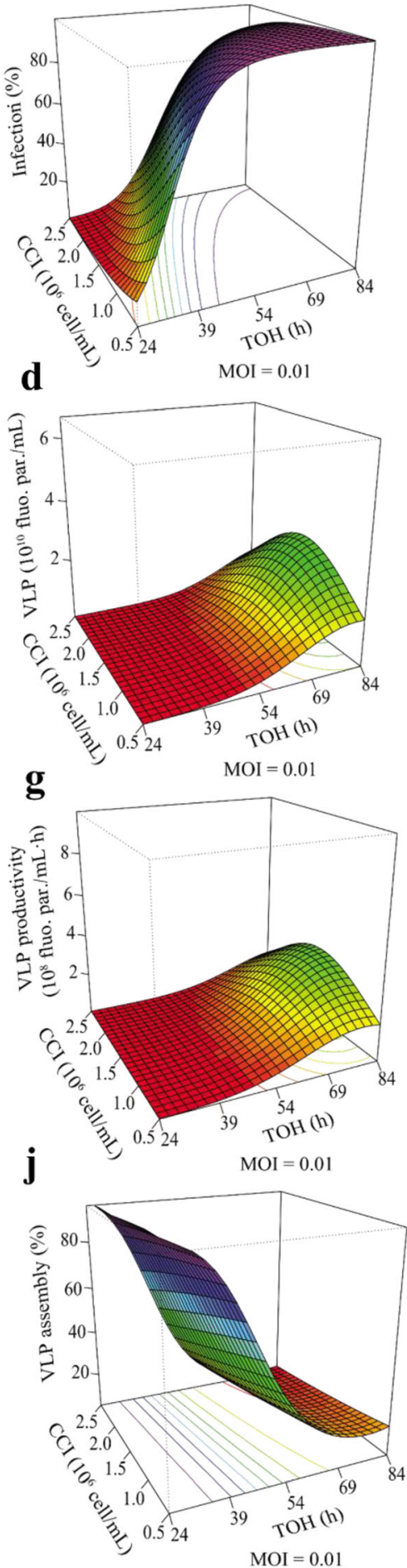

b

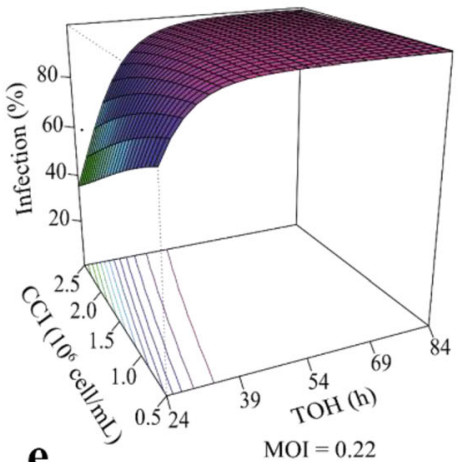

e

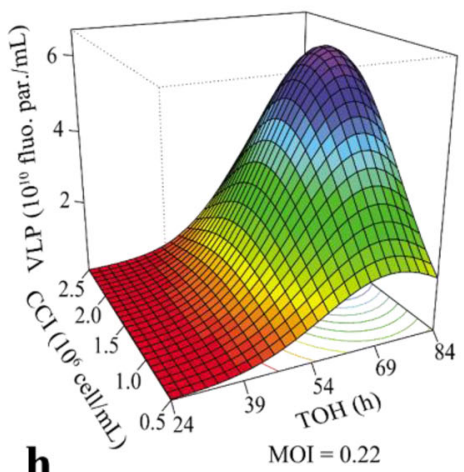

h

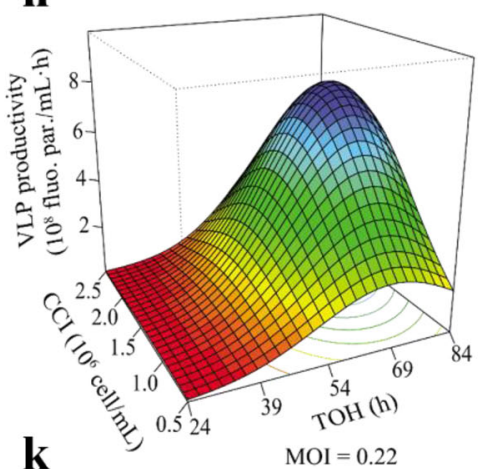

$\mathbf{k}$

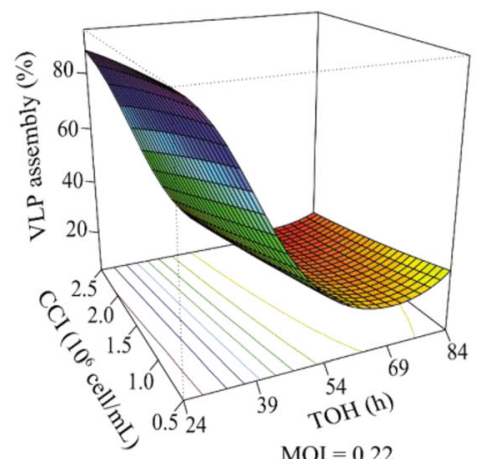

c

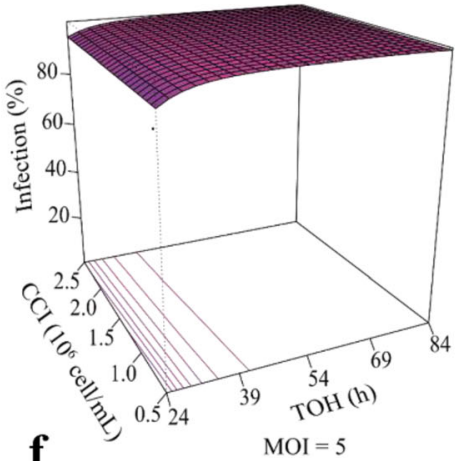

f

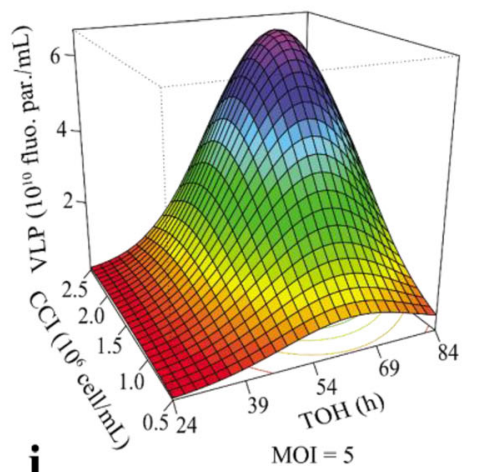

i
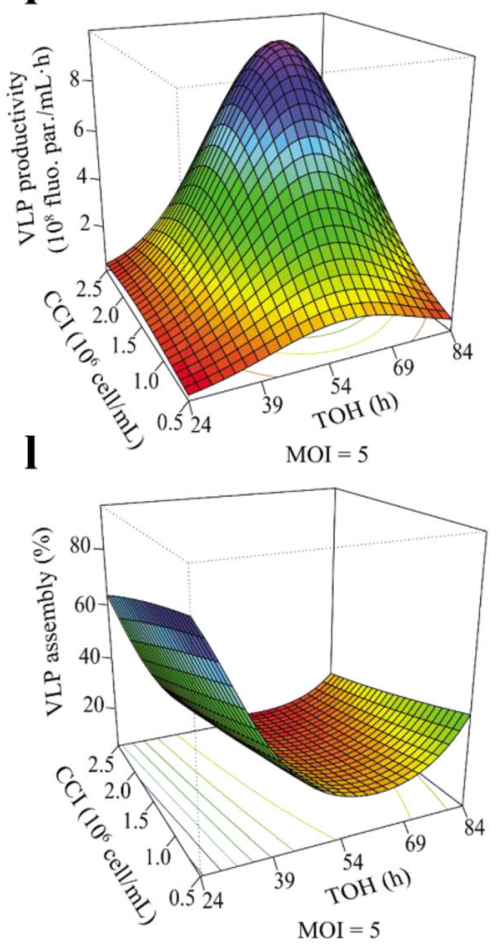

Fig. 2 Three-dimensional plots of the different functions based on the Box-Behnken experimental results. a-c Baculovirus infection. d-f VLP production. $\mathbf{g}-\mathbf{i}$ VLP productivity. $\mathbf{j}-\mathbf{I}$ VLP assembly as a function of CCI,

decreased the ratio of VLPs to non-assembled monomers, regardless the value of CCI.

The models were used to predict an optimal combination of $\mathrm{CCI}, \mathrm{MOI}$ and $\mathrm{TOH}$ to maximize each specific response. In
MOI, and TOH. All the graphs were constructed by representing the effect of two independent variables on a response while maintaining the third one at a fixed level this case, different independent variable combinations resulted in the optimum condition for each function (Table 2), thus making difficult to define a global optimum condition combining all the different models at once. Therefore, a multiple- 
Table 2 Optimal CCI, MOI, and TOH conditions maximizing each response function

\begin{tabular}{llll}
\hline Response function & CCI $\left(10^{6}\right.$ cell $\left./ \mathrm{mL}\right)$ & MOI (pfu/cell) & TOH (h) \\
\hline Infection efficiency & 0.5 & 0.2 & 76.4 \\
VLP production & 2.0 & 1.3 & 73.5 \\
VLP assembly & 0.5 & 0.01 & 24.0 \\
VLP productivity & 2.0 & 2.3 & 67.8 \\
\hline
\end{tabular}

criteria decision analysis (MCDA) based on desirability functions was applied in order to determine a global optimum combining the different functions. Every model function was transformed to a dimensionless scale (Eq. 4) and given a weight according to the importance of each response. A higher priority was assigned to VLP production, VLP assembly, and VLP productivity $(w=2)$, while a lower restriction was assigned to infection $(w=1)$. Two possible response combinations were considered in the MCDA, the first focused on maximizing product quantity and the second one maximizing product quality. To do this, the VLP assembly function was excluded from the MCDA in the definition of a global optimal condition targeting product quantity. The application of the MCDA for the product quality optimum resulted in a CCI, MOI, and TOH of $1.1 \times 10^{6}$ cell $/ \mathrm{mL}, 1 \mathrm{pfu} / \mathrm{cell}$, and $33.6 \mathrm{hpi}$, respectively. The optimum maximizing product quantity was determined as $2.0 \times 10^{6} \mathrm{cell} / \mathrm{mL}$ for $\mathrm{CCI}$, a MOI of $2.5 \mathrm{pfu} / \mathrm{cell}$, and a TOH of $69.3 \mathrm{hpi}$.

\section{Validating the quantity and quality global optima}

The two global optimal conditions obtained by the combination of RSM and MCDA were successfully corroborated in a validation experiment. High Five cell growth was arrested after BV-GageGFP infection with $>80 \%$ of the culture infected at 24 hpi (Fig. 3a-b). As expected, a higher cell viability was observed for the quality optima (33.6 hpi) compared with the quantity condition at TOH (69.3 hpi). Confocal microscopy analysis of infected cells showed successful colocalization (yellow) of the GageGFP polyprotein (green) with the cell membrane (red), thus indicating that VLP formation and production was taking place (Fig. 3c). This process could be tracked in real time using a confocal fluorescence microscope with the high-speed acquisition mode (Fig. S1).

Measurement of fluorescence indicated that the highest pace of GageGFP production was achieved with higher MOI. Also, the highest fluorescence yield in the supernatant was achieved with the quantity optimum $(2448.6 \pm 23.8 \mathrm{RFU})$ at $69.3 \mathrm{hpi}$ (Fig. 4a). The TOH for the quality optimum condition coincided with the peak of intracellular GageGFP production, but not for the quantity optimum. In all cases, GageGFP production in the supernatant progressively increased with longer $\mathrm{TOH}$, although the intracellular
GageGFP levels reached a maximum concentration that subsequently decreased (Fig. 4b). Analysis of the VLP production process of the quantity optimum by flow virometry evidenced that there was a continuous increase in VLP concentration (Fig. 4c). Comparison of VLP production in both optima by means of nanoparticle tracking analysis (NTA) yielded a 10fold and 4.8-fold increase in production and productivity in the quantity over the quality optimum, respectively. In all cases, the experimental outcome was within the predicted limits (Table 3). Similar ratios of VLPs to total nanoparticles were observed, representing a $9.7 \pm 3.1 \%$ for the quality and a $9.3 \pm 1.1 \%$ for the quantity condition. In contrast, analysis of the optima by sucrose cushion ultracentrifugation showed a 4.9-fold increase of GageGFP monomer assembled in the form of VLPs in the quality optimum $(64.6 \pm 5.1 \%)$. The SN and $\mathrm{SN}-25 \%$ fractions, corresponding to non-assembled GageGFP, showed significantly larger levels of fluorescence in the quantity optimum (Fig. 4d). However, VLP-containing fractions (25-45\% and 45\%) were more enriched in the quality condition. BV-GageGFP titration of both optima by plaque assay resulted in a 2.7-fold increase of infectious particles in the quantity $\left(13.2 \pm 5.1 \times 10^{7} \mathrm{pfu} / \mathrm{mL}\right)$ compared with quality condition $\left(4.8 \pm 1.5 \times 10^{7} \mathrm{pfu} / \mathrm{mL}\right)$.

An acceptable correlation between indirect VLP quantification with spectrofluorometry and direct nanoparticle measurement with NTA was only achieved for the quality optimum condition (Eq. 1). On the contrary, GageGFP fluorescence levels of the quantity optimum measured by spectrofluorometry (2448.6 $\pm 23.8 \mathrm{RFU})$ and their conversion to VLP concentration according to Eq. 1 resulted in a 9.6-fold higher value than the one obtained by direct VLP quantification using NTA. However, multiplying this fluorescence value by the VLP assembly factor calculated by analytical ultracentrifugation (Table 3) allowed to determine the GageGFP fluorescence exclusively attributed to VLPs $(323.2 \pm 3.1 \mathrm{RFU})$ and discard that of unassembled GageGFP monomer. Introducing the fluorescence value associated to VLPs in Eq. 1 resulted in a better prediction to NTA quantification $\left(4.9 \pm 0.5 \times 10^{10}\right.$ $\mathrm{VLP} / \mathrm{mL}$ ). Supernatant characterization of the quality and quantity optima by cryogenic transmission electron microscopy (cryo-TEM) indicated that VLPs were correctly formed in both conditions (Fig. 5). Interestingly, the median VLP size was higher in the quality $(181.4 \pm 4.4 \mathrm{~nm})$ compared with the quantity optimum $(164.7 \pm 0.3 \mathrm{~nm})$. The presence of EVs and baculoviruses was also detected, in agreement with the existence of a non-fluorescent particle population observed with NTA.

\section{Comparison with other VLP production conditions}

In addition to the optimization work performed, the quantity optimum condition was compared with two conditions used in other works for the production of high VLP titers in order to 

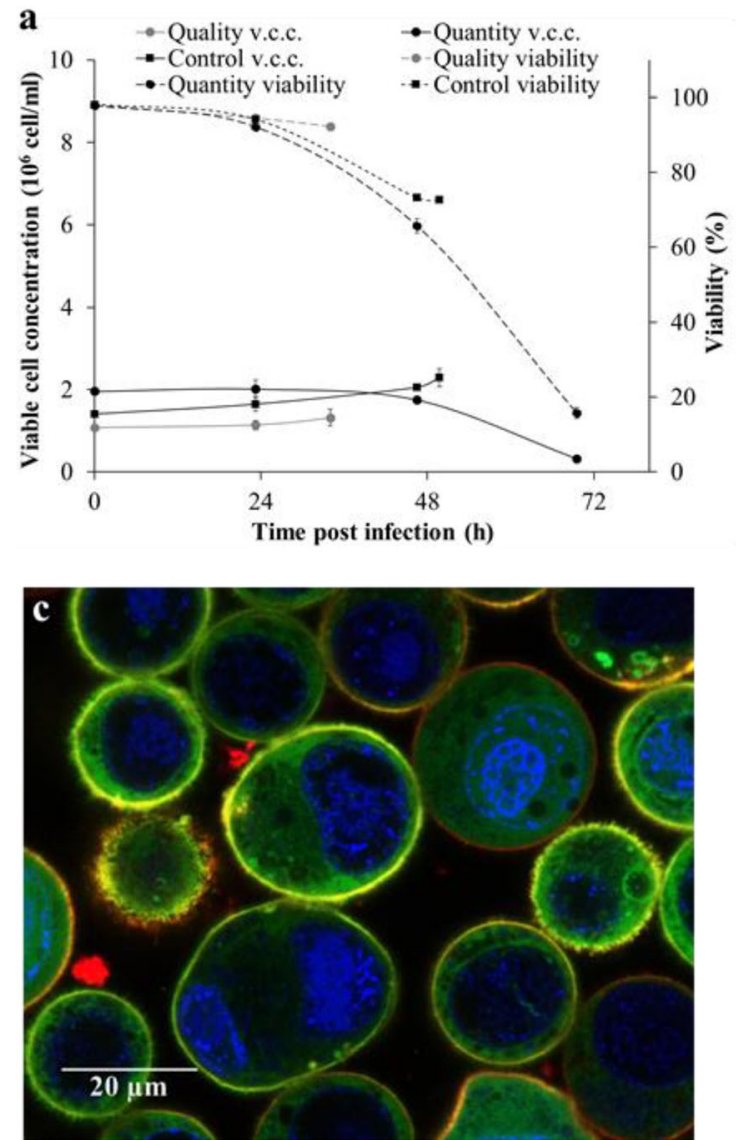

Fig. 3 Validation experiment of the quality and quantity optima. a Viable cell concentration and viability progression after infection with the BVGageGFP. The center point of the Box-Behnken DoE (infection control) was also included to account for the robustness of the infection process, with cells infected with a MOI of 0.22 at $1.5 \times 10^{6}$ cell $/ \mathrm{mL}$ and harvested at $54 \mathrm{hpi}$. b BV-GageGFP infection process in the different conditions

corroborate the results of the study. The first approach consisted in infecting High Five cells at a high cell concentration and MOI. To this purpose, High Five cells were infected at $6 \times 10^{6} \mathrm{cell} / \mathrm{mL}$ with a MOI of 5 . The second strategy consisted in performing a medium replacement before infection using the quantity optimum. This option was considered as a means to value if the added effort of performing a medium replacement would provide a substantial advantage in VLP concentration. Both conditions were not examined in the DoE in order to avoid the added difficulties required at process level, but were compared with the quantity optimum as a contrast since they have been used in the literature. Baculovirus infection progressed more rapidly after medium exchange than in the original condition but the drop in cell viability was not significantly affected (Fig. 6a-b). On the other hand, baculovirus infection advanced more slowly in the high CCI condition, to the point that cell culture was not fully infected after $72 \mathrm{hpi}$. Medium exchange before infection increased GageGFP production by 1.3 -fold at $69.3 \mathrm{hpi}$, b

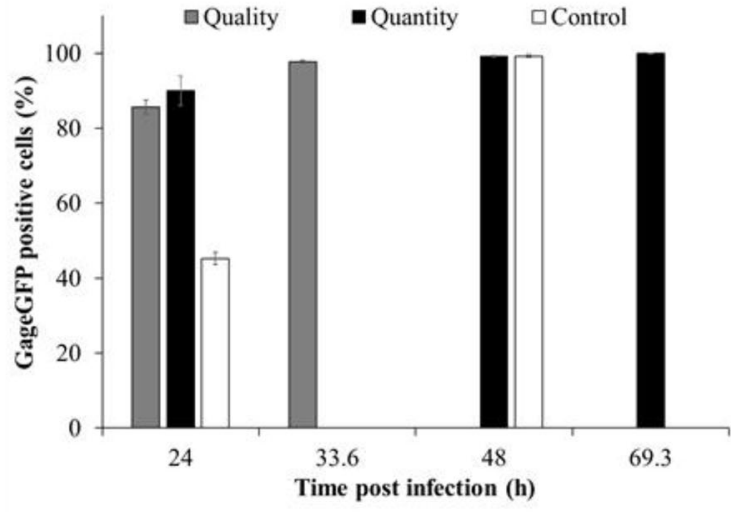

tested. Infected cells were measured every $24 \mathrm{~h}$ until their optimal TOH. c High Five cells infected with the BV-GageGFP at $2.0 \times 10^{6} \mathrm{cell} / \mathrm{mL}$ and a MOI of 2.5 and observed at $48 \mathrm{hpi}$. Cell nucleus was stained with Hoechst (blue) and cell membrane with CellMask ${ }^{\mathrm{TM}}$ (red). The mean and standard deviation of triplicate experiments are represented

corresponding to $3168.6 \pm 100.2 \mathrm{RFU}$ in comparison with the $2528.0 \pm 76.3 \mathrm{RFU}$ achieved in the quantity condition (Fig. 6c-d). The same improvement was observed in VLP quantification by NTA, with $4.3 \pm 0.2 \times 10^{10} \mathrm{VLP} / \mathrm{mL}$ for medium exchange while $3.4 \pm 0.3 \times 10^{10} \mathrm{VLP} / \mathrm{mL}$ was measured for the quantity optimum condition. However, baculovirus infection at the high CCI condition reached a significantly lower GageGFP production $(66.6 \pm 3.3 \mathrm{RFU})$ and also in terms of VLP production $\left(2.3 \pm 0.6 \times 10^{8} \mathrm{VLP} / \mathrm{mL}\right)$.

The median size of the VLPs produced in these conditions did not substantially vary from the quantity optimum (168.2 \pm $3.3 \mathrm{~nm}$ ), being $169.6 \pm 3.3 \mathrm{~nm}$ for the medium exchange and $160.5 \pm 3.7 \mathrm{~nm}$ for the high CCI. Similar levels of total nanoparticles were obtained in the three different conditions, thus representing a big difference in the ratio of VLP to total nanoparticles (Table 4). Remarkable differences were also observed as regards the amount of infectious baculovirus particles produced. Medium replacement before infection increased the levels by 2.1 -fold $\left(47.0 \pm 9.9 \times 10^{7} \mathrm{pfu} / \mathrm{mL}\right)$ compared with 

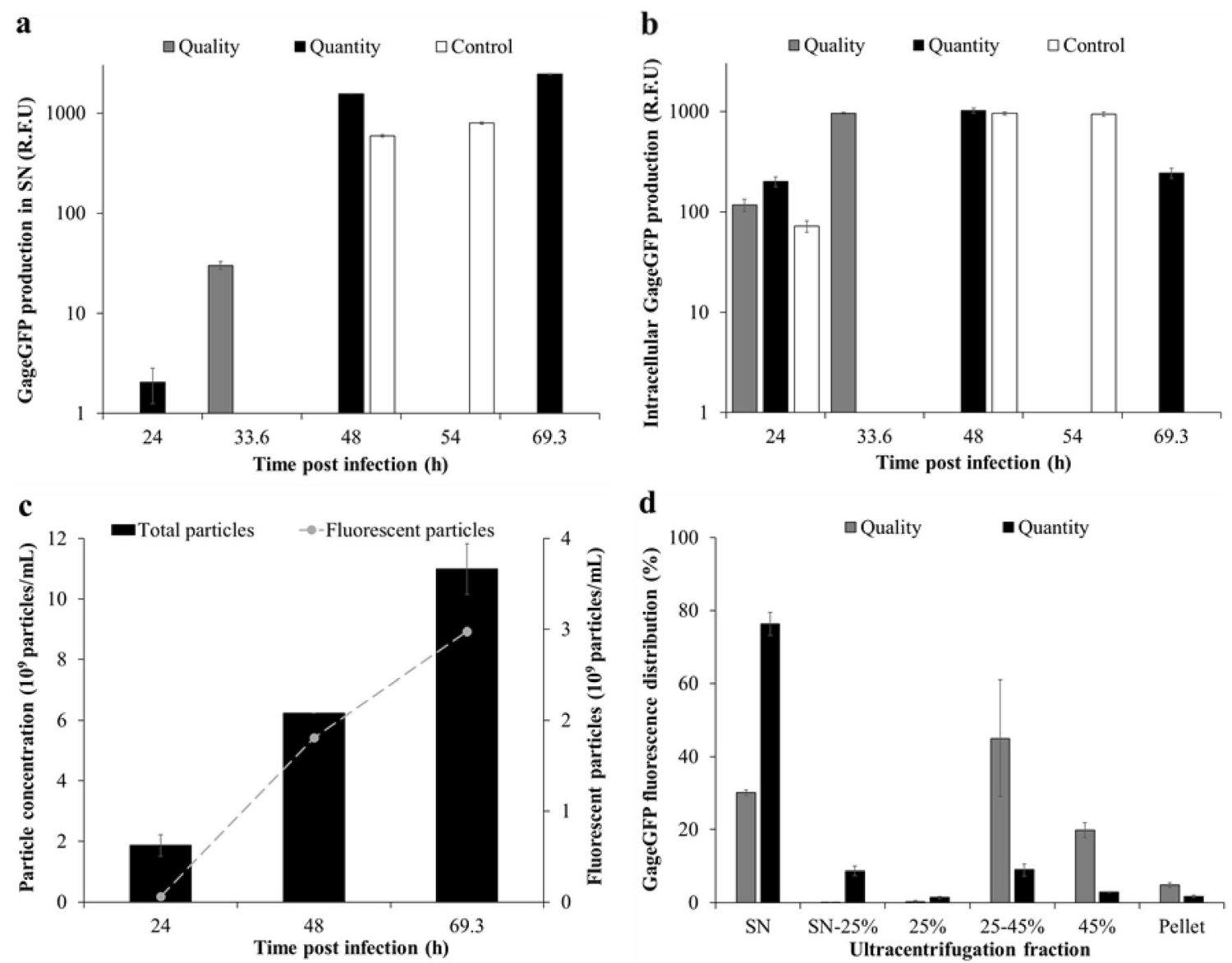

Fig. 4 GageGFP and VLP production in the validation experiment. a-b GageGFP production process in the supernatant and intracellularly. Samples were taken every $24 \mathrm{~h}$ until their respective TOH. c Fluorescent and total nanoparticle production profile measured by flow

virometry in the quantity optimum condition. $\mathbf{d}$ Fluorescence distribution after double sucrose cushion ultracentrifugation of the quality and quantity optima. These experiments were performed in triplicate

the $22.7 \pm 2.5 \times 10^{7} \mathrm{pfu} / \mathrm{mL}$ achieved in the quantity optimum condition. However, a much lower baculovirus titer of $0.9 \pm$ $0.3 \times 10^{7} \mathrm{pfu} / \mathrm{mL}$ was obtained in the high CCI condition.

\section{Discussion}

The High Five/BEVS has demonstrated a high versatility to produce a wide variety of recombinant proteins. However, the capabilities of the High Five cell line as a production platform

of complex multimeric structures, such as enveloped VLPs, have not been explored in detail. To date, the assessment of VLP production has been performed based on indirect quantification methods (Pillay et al. 2009). Moreover, most of the research has been conducted either in low performing or animal compound containing cell culture media (Krammer et al. 2010; Wilde et al. 2014). Although these works have brought advances in the production of nanoparticles using the High Five/BEVS, a deeper study would contribute to exploit the full potential of such platform.

Table 3 Experimental validation of the global optimal conditions and comparison with model predictions. VLP concentration measurements were carried out with NTA. The prediction intervals represent the lower and the upper limits according to the logarithmic or logistic response transformation

\begin{tabular}{lcccccc}
\hline \multirow{2}{*}{ Response } & \multicolumn{2}{c}{ Product quality } & & & \multirow{2}{*}{ Product quantity } \\
\cline { 2 - 3 } \cline { 5 - 6 } & Experimental & Model prediction & & Experimental & Model prediction \\
\hline Infection $(\%)$ & $97.8 \pm 2.0$ & {$[71.6-99.7]$} & & 100 & {$[96.6-100]$} \\
VLP production $\left(10^{8}\right.$ fluorescent particle $\left./ \mathrm{mL}\right)$ & $39.0 \pm 4.5$ & {$[15.6-60.2]$} & & $391.0 \pm 13.9$ & {$[337.2-1333.4]$} \\
VLP assembly $(\%)$ & $64.6 \pm 5.1$ & {$[46.4-72.9]$} & & $13.2 \pm 1.0$ & {$[5.7-18.2]$} \\
VLP productivity $\left(10^{6}\right.$ fluorescent particle $\left./ \mathrm{mL} \mathrm{h}\right)$ & $118.1 \pm 27.8$ & {$[48.3-186.2]$} & & $564.2 \pm 20.2$ & {$[477.6-1884.8]$} \\
\hline
\end{tabular}



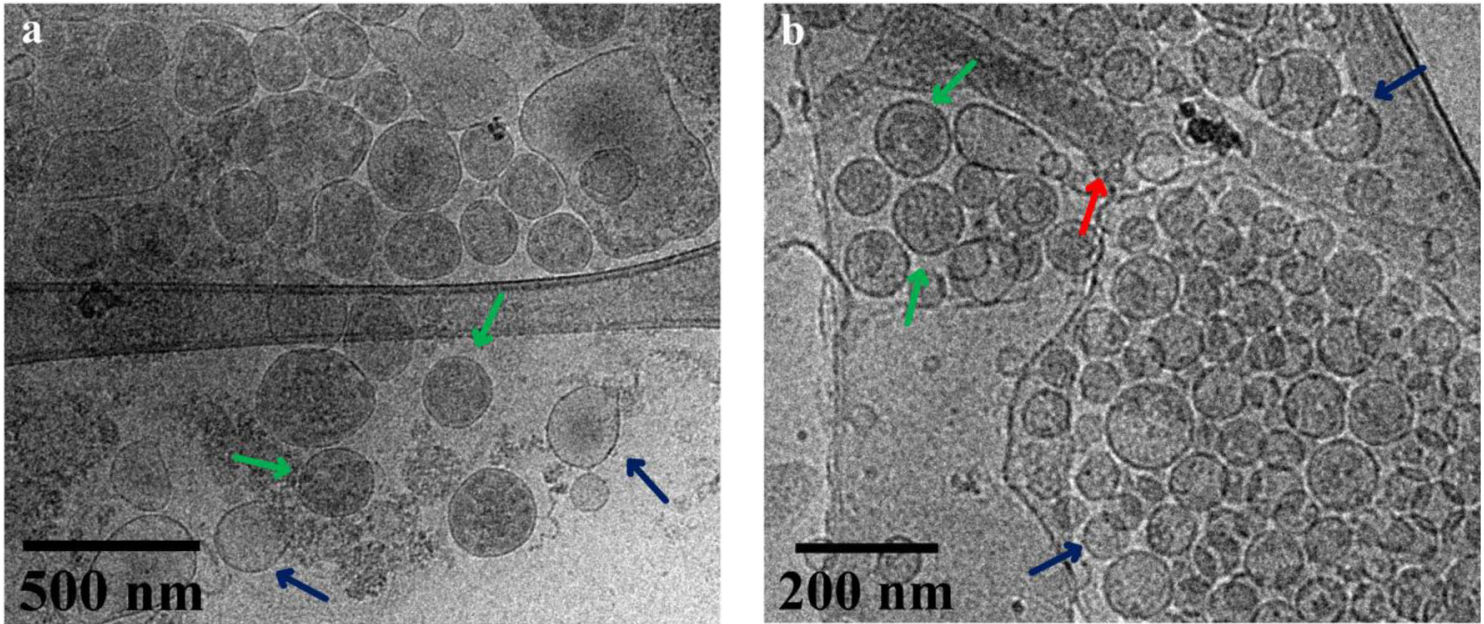

Fig. 5 GageGFP VLP characterization by cryo-TEM. a-b Supernatant visualization of the quality (33.6 hpi) and quantity (69.3 hpi) optima, respectively. The arrows indicate the different specimens: VLP (green), EV (blue), and baculovirus (red)

$\mathrm{CCI}, \mathrm{MOI}$, and $\mathrm{TOH}$ were selected as the critical parameters affecting the production of HIV-1 Gag VLPs in the BEVS. For a comprehensive analysis, four different responses were considered and simultaneously evaluated by means of RSM and MCDA. TOH and MOI were shown to be particularly relevant to achieve a high VLP concentration but also in

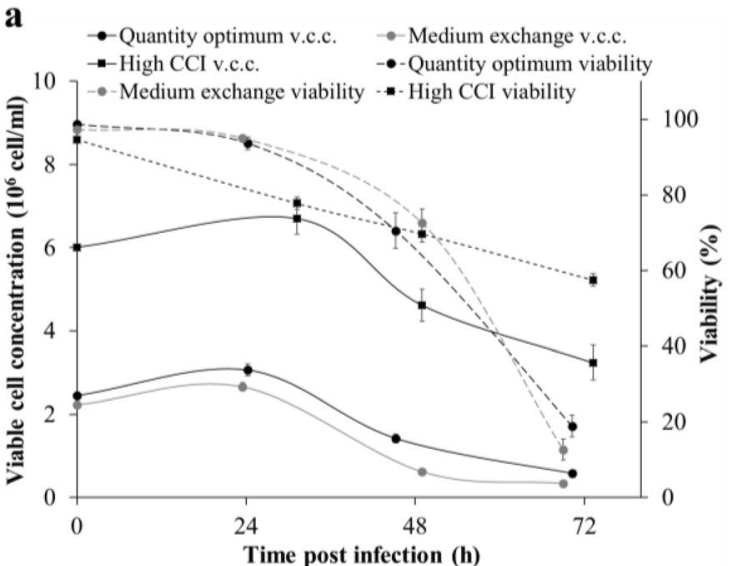

b
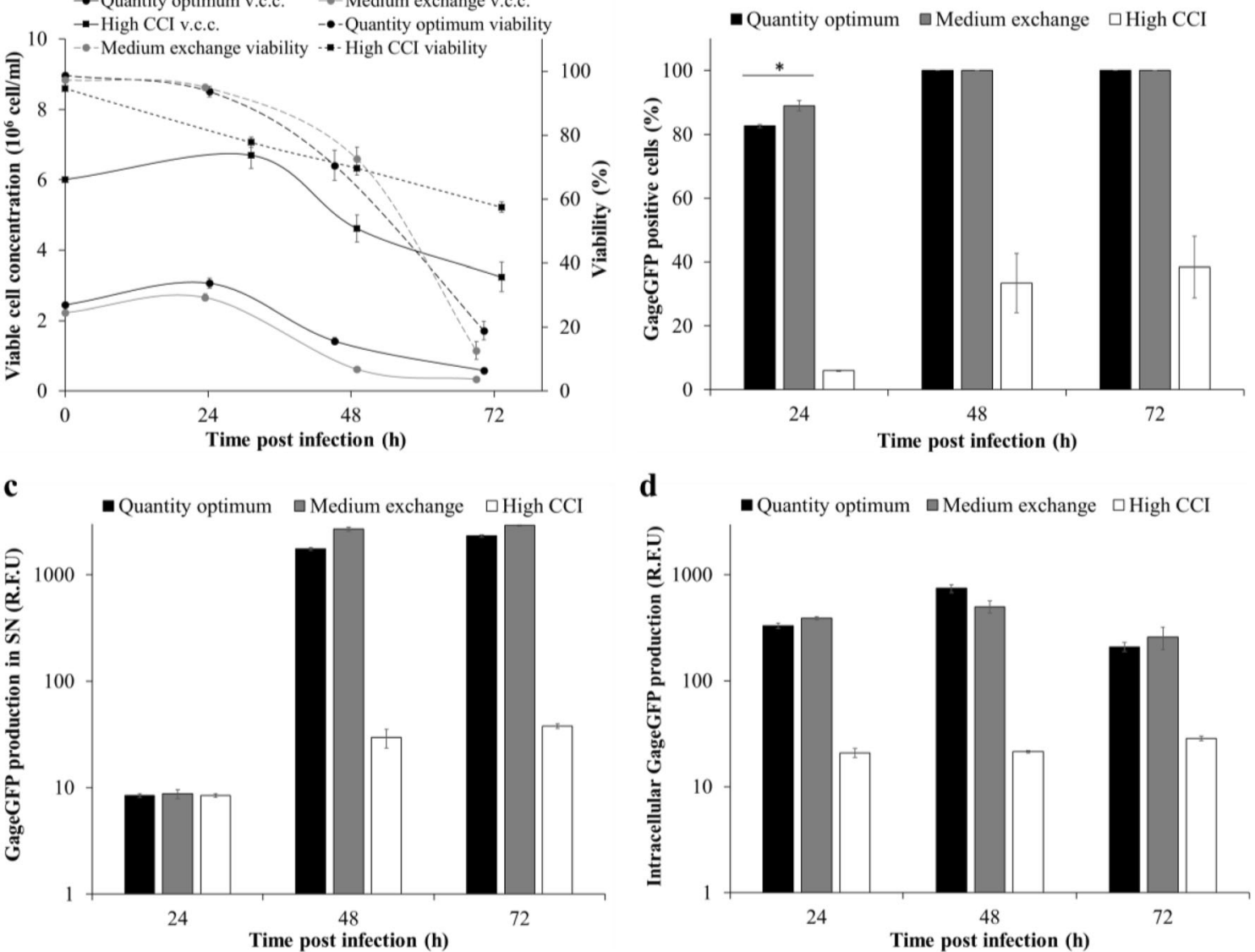

Fig. 6 Comparison of VLP production using the quantity optimum with medium exchange and baculovirus infection at a high CCI. a-b Viable cell concentration, cell viability, and BV-GageGFP infection process in the different conditions tested. $\mathbf{c}-\mathbf{d}$ Supernatant and intracellular GageGFP production. The average of triplicate experiments is

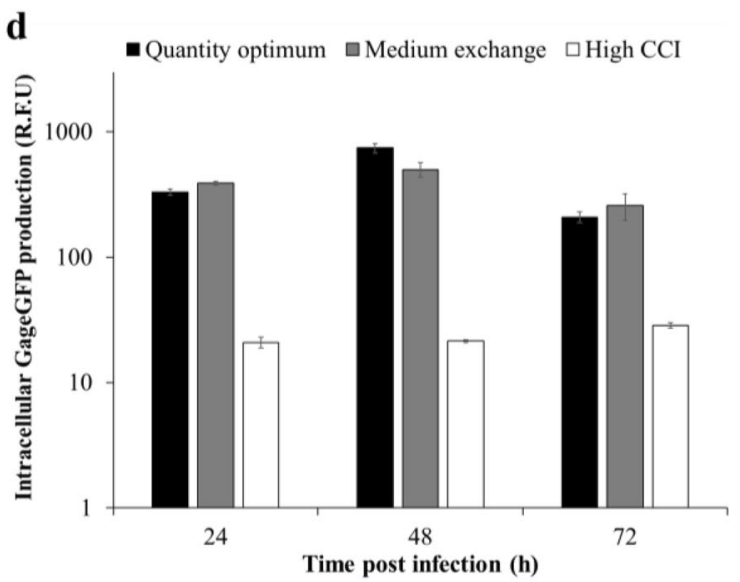

represented. Medium exchange was performed prior to BV-GageGFP infection and consisted in infecting High Five cells at $2.0 \times 10^{6}$ cell $/ \mathrm{mL}$ with a MOI of $2.5 \mathrm{pfu} / \mathrm{cell}$. As regards the high CCI condition, High Five cells were infected at $6 \times 10^{6} \mathrm{cell} / \mathrm{mL}$ with a MOI of 5 
Table 4 Comparison of GageGFP VLP production levels of the quantity optimum, medium exchange, and high CCI conditions measured by NTA

\begin{tabular}{lllll}
\hline Condition & $\begin{array}{l}\text { VLP production } \\
\left(10^{8} \text { fluorescent particles } / \mathrm{mL}\right)\end{array}$ & $\begin{array}{l}\text { Total particle production } \\
\left(10^{8} \text { particles } / \mathrm{mL}\right)\end{array}$ & $\begin{array}{l}\text { Ratio fluorescent particles/ } \\
\text { total particles }(\%)\end{array}$ & VLP size (nm) \\
\hline Quantity optimum & $335.0 \pm 29.0$ & $4260.0 \pm 609.0$ & $8 \pm 1.7$ & $168.2 \pm 3.3$ \\
Medium exchange & $432.0 \pm 22.0$ & $5510.0 \pm 232.0$ & $7.8 \pm 0.2$ & $169.6 \pm 3.3$ \\
High CCI & $2.3 \pm 0.6$ & $5230.0 \pm 767.0$ & 0.1 & $160.5 \pm 3.7$ \\
\hline
\end{tabular}

maintaining an adequate ratio of GageGFP assembly in the form of VLPs. Pillay and co-workers used a factorial experiment to assess the effect of different MOI comprised in the range of 0.1-5 in High Five cells (Pillay et al. 2009). They did not identify any significant difference in Gag yield, but here we observed that MOI plays a significant role in VLP production. Interestingly, including a function for VLP assembly based on the measurement of the GageGFP fluorescence distribution in the different fractions of an ultracentrifugation proved to be highly relevant. These results in combination with direct nanoparticle quantification by NTA and flow virometry permitted to obtain a more accurate perspective of the process.

A pronounced decrease in VLP assembly was shown with increasing $\mathrm{TOH}$, limiting a favorable combination with the other three responses. To this purpose, two different global optimization strategies were examined by MCDA, one considering the VLP assembly function (therefore with priority on product quality) and the other not (therefore with priority on product quantity). Remarkable differences were encountered between the two optima, achieving a 10-fold higher VLP production in the quantity condition, whereas a 4.9 -fold increase in the assembly of GageGFP as VLPs was obtained in the quality optimum. Then, it is evident that High Five cells possess a high capacity to produce GageGFP, but there is a remarkable competition between the formation of VLPs and the release of unassembled GageGFP monomer. The large amount of GageGFP produced, potentially overcoming the capacity of the cell line itself to process all the GageGFP in the form of VLPs, combined to cell membrane disruption could be the reason explaining these results. This could be appreciated even in the quality optimum itself, in which a high cell viability of $92.4 \pm 0.6 \%$ was measured at harvest (33.6 hpi) (Fig. 3a) and a $64.6 \pm 5.1 \%$ of the fluorescence in the supernatant was associated to VLPs, meaning that around the $35 \%$ remaining was due to unassembled GageGFP monomer. These results emphasize the need to complement the methods most currently used for VLP identification or quantification based on monomer detection with direct nanoparticle assessment techniques, specially using the High Five cell line (Maranga et al. 2003; Pillay et al. 2009; Tagliamonte et al. 2010; Shoja et al. 2013; Monteiro et al. 2016; Fuenmayor et al. 2019).
The application of cryo-TEM helped to gain insight into the identification of the morphological features of the VLPs produced (Fig. 5). Cryo-TEM has recently been highlighted as powerful methodology for nanoparticle characterization as the different specimens can be observed in native conditions (Martin et al. 2016; Maldonado et al. 2016; Gallagher et al. 2018). GageGFP VLPs presented the expected size of 100 $200 \mathrm{~nm}$ and morphology consistent with immature HIV-1 VLPs, comparable with VLPs produced in Sf9 (Nika et al. 2017) and HEK 293 cells (Venereo-Sanchez et al. 2016). The rod-shaped structure corresponding to the infective form of the baculovirus was also detected. The presence of EVs was observed alongside VLPs, which are known to be produced as way of communication in eukaryotic cells (Ratajczak et al. 2006). EVs were more heterogeneous in size $(50-500 \mathrm{~nm})$ and shape compared with VLPs and less electron-dense. These results coincide with the measurements performed by NTA and flow virometry where a remarkable population of non-fluorescent nanoparticles was detected. Future efforts are needed to understand their role in insect cell lines since little information is available.

A maximum HIV-1 GageGFP VLP concentration of $3.9 \pm$ $0.1 \times 10^{10} \mathrm{VLP} / \mathrm{mL}$ was achieved in the supernatant using the product quantity optimum. Thus, these conditions would be selected in case of designing a VLP manufacturing process. However, if the presence of baculoviruses posed a risk for the final VLP application, the quality optimum provides a 2.7-fold reduction in infectious particles, which would facilitate the downstream process. Difficulties were encountered in the comparison of the VLP titer obtained since most of the studies producing Gag VLPs with the insect cell/BEVS used indirect quantification methods based on monomer detection (Buonaguro et al. 2001; Pillay et al. 2009; Visciano et al. 2011). Nevertheless, when the comparison of nanoparticle production was possible, this optimum condition proved to be superior to Gag VLP production in HEK 293 cells without (Cervera et al. 2013) and with additive supplementation (Cervera et al. 2015a) by 14.4 and 1.7-fold, respectively. Moreover, these conditions proved to be superior to influenza VLP production using the Sf9 (Thompson et al. 2015) and High Five/BEVS (Carvalho et al. 2016) by 6.7 and 25 -fold, respectively. The potential of the quantity optimum was 
also evaluated against other baculovirus infection strategies in this work. Infection at a high CCI using a high MOI resulted in a much lower yield of VLP production probably owing to the scarcity of some nutrients in the medium (Table 4). BV production was also significantly reduced in such conditions. These differences in production could be related to the cell density effect previously described in insect cells with the BEVS (Bernal et al. 2009; Huynh et al. 2013; Huynh et al. 2014). On the other side, medium replacement before $\mathrm{BV}$ infection moderately increased VLP production but duplicated baculovirus production. This strategy has proven to be an efficient manner to increase both the yield of recombinant proteins (Caron et al. 1990) and baculoviruses (Carinhas et al. 2009) with the Sf9/ BEVS cells and also in mammalian cells (Bollin et al. 2011; Cervera et al. 2015b). In this case, no substantial benefit was obtained, thus indicating that the added efforts required to perform medium replacement might not be advantageous.

In conclusion, a detailed study of Gag VLP production using the High Five/BEVS was conducted. The application of RSM and MCDA allowed to go a step further and capture the synergies between the influential factors affecting the VLP production process. The combination of advanced statistical methods with a variety of nanoparticle quantification and characterization techniques allowed to obtain a more comprehensive picture of the process and to determine a highyielding and reproducible condition for VLP production. The approach here proposed is valuable for studies dealing with the production of other complex nanoparticles. Future efforts in transferring this process into bioreactor scale should allow to define a robust platform for VLP production.

Acknowledgments The authors would like to thank Dr. Paula Alves (Instituto de Biologia Experimental e Tecnológica, Oeiras, Portugal) and Dr. Nick Berrow (Institute for Research in Biomedicine, Barcelona, Spain) for providing the High Five and Sf9 cell lines, respectively. Marta Martínez-Calle developed the BV-GageGFP. Ángel Calvache and Jorge Fomaro (Beckman Coulter) facilitated the access to the CytoFlex LX flow cytometer. The support of Martí de Cabo and Mónica Roldán from Servei de Microscòpia of Universitat Autònoma de Barcelona (UAB) with the cryo-TEM and confocal microscopy, respectively, and Paolo Saccardo from Plataforma de Producción de Proteínas, CIBER de Bioingeniería, Biomateriales y Nanomedicina (CIBER-BBN) of UAB with baculovirus titration are appreciated. The research group is recognized as 2017 SGR 898 by Generalitat de Catalunya.

Funding information Eduard Puente-Massaguer is a recipient of an FPU grant from Ministerio de Educación, Cultura y Deporte of Spain (FPU15/ 03577).

\section{Compliance with ethical standards}

Conflict of interest The authors declare that they have no competing interests.

Ethical approval This article does not contain any studies with human participants performed by any of the authors.

\section{References}

Andersson AMC, Schwerdtfeger M, Holst PJ (2018) Virus-like-vaccines against HIV. Vaccines 6:1-17

Barbur VA, Montgomery DC, Peck EA (1994) Introduction to linear regression analysis. Wiley

Bernal V, Carinhas N, Yokomizo AY, Carrondo MJT, Alves PM (2009) Cell density effect in the baculovirus-insect cells system: a quantitative analysis of energetic metabolism. Biotechnol Bioeng 104: $162-180$

Bollin F, Dechavanne V, Chevalet L (2011) Design of experiment in CHO and HEK transient transfection condition optimization. Protein Expr Purif 78:61-68

Bukzem AL, Signini R, dos Santos DM, Lião LM, Ascheri DPR (2016) Optimization of carboxymethyl chitosan synthesis using response surface methodology and desirability function. Int J Biol Macromol 85:615-624

Buonaguro L, Buonaguro FM, Tornesello ML, Mantas D, Beth-Giraldo E, Wagner R, Michelson S, Prevost MC, Wolf H, Giraldo G (2001) High efficient production of Pr55gag virus-like particles expressing multiple HIV-1 epitopes, including a gp120 protein derived from an Ugandan HIV-1 isolate of subtype A. Antivir Res 49:35-47

Buonaguro L, Tagliamonte M, Visciano ML (2013) Chemokine receptor interactions with virus-like particles. In: Methods in molecular biology. Humana Press, Totowa, pp 57-66

Carinhas N, Bernal V, Yokomizo AY, Carrondo MJT, Oliveira R, Alves PM (2009) Baculovirus production for gene therapy: the role of cell density, multiplicity of infection and medium exchange. Appl Microbiol Biotechnol 81:1041-1049

Caron AW, Archambault J, Massie B (1990) High-level recombinant protein production in bioreactors using the baculovirus-insect cell expression system. Biotechnol Bioeng 36:1133-1140

Carvalho SB, Freire JM, Moleirinho MG, Monteiro F, Gaspar D, Castanho MARB, Carrondo MJT, Alves PM, Bernardes GJL, Peixoto C (2016) Bioorthogonal strategy for bioprocessing of specific-site-functionalized enveloped influenza-virus-like particles. Bioconjug Chem 27:2386-2399

Cervera L, Gutiérrez-Granados S, Martínez M, Blanco J, Gòdia F, Segura MM (2013) Generation of HIV-1 Gag VLPs by transient transfection of HEK 293 suspension cell cultures using an optimized animal-derived component free medium. J Biotechnol 166:152-165

Cervera L, Fuenmayor J, González-Domínguez I, Gutiérrez-Granados S, Segura MM, Gòdia F (2015a) Selection and optimization of transfection enhancer additives for increased virus-like particle production in HEK293 suspension cell cultures. Appl Microbiol Biotechnol 99:9935-9949

Cervera L, Gutiérrez-Granados S, Berrow NS, Segura MM, Gòdia F (2015b) Extended gene expression by medium exchange and repeated transient transfection for recombinant protein production enhancement. Biotechnol Bioeng 112:934-946

Deo VK, Kato T, Park EY (2015) Chimeric virus-like particles made using GAG and M1 capsid proteins providing dual drug delivery and vaccination platform. Mol Pharm 12:839-845

Fernandes F, Teixeira AP, Carinhas N, Carrondo MJT, Alves PM (2013) Insect cells as a production platform of complex virus-like particles. Expert Rev Vaccines 12:225-236

Fuenmayor J, Cervera L, Gòdia F, Kamen A (2019) Extended gene expression for Gag VLP production achieved at bioreactor scale. J Chem Technol Biotechnol 94:302-308

Gallagher J, McCraw D, Torian U, Gulati N, Myers M, Conlon M, Harris A (2018) Characterization of hemagglutinin antigens on influenza virus and within vaccines using electron microscopy. Vaccines 6:121 
Gómez-Sebastián S, López-Vidal J, Escribano JM (2014) Significant productivity improvement of the baculovirus expression vector system by engineering a novel expression cassette. PLoS One 9:1-10

Gutiérrez-Granados S, Cervera L, Gòdia F, Carrillo J, Segura MM (2013) Development and validation of a quantitation assay for fluorescently tagged HIV-1 virus-like particles. J Virol Methods 193:85-95

Gutiérrez-Granados S, Cervera L, Segura M d 1 M, Wölfel J, Gòdia F (2016) Optimized production of HIV-1 virus-like particles by transient transfection in CAP-T cells. Appl Microbiol Biotechnol 100: 3935-3947

Haynes JR, Dokken L, Wiley JA, Cawthon AG, Bigger J, Harmsen AG, Richardson C (2009) Influenza-pseudotyped Gag virus-like particle vaccines provide broad protection against highly pathogenic avian influenza challenge. Vaccine 27:530-541

Hermida-Matsumoto L, Resh MD (2000) Localization of human immunodeficiency virus type $1 \mathrm{Gag}$ and Env at the plasma membrane by confocal imaging. J Virol 74:8670-8679

Honary S, Ebrahimi P, Hadianamrei R (2014) Optimization of particle size and encapsulation efficiency of vancomycin nanoparticles by response surface methodology. Pharm Dev Technol 19:987-998

Huynh HT, Tran TTB, Chan LCL, Nielsen LK, Reid S (2013) Decline in baculovirus-expressed recombinant protein production with increasing cell density is strongly correlated to impairment of virus replication and mRNA expression. Appl Microbiol Biotechnol 97:5245-5257

Huynh HT, Tran TTB, Chan LCL, Nielsen LK, Reid S (2014) Effect of the peak cell density of recombinant AcMNPV-infected Hi5 cells on baculovirus yields. Appl Microbiol Biotechnol 99:1687-1700

Krammer F, Schinko T, Palmberger D, Tauer C, Messner P, Grabherr R (2010) Trichoplusia ni cells (High Five ${ }^{\mathrm{TM}}$ ) are highly efficient for the production of influenza A virus-like particles: a comparison of two insect cell lines as production platforms for influenza vaccines. Mol Biotechnol 45:226-234

Maldonado J, Cao S, Zhang W, Mansky L (2016) Distinct morphology of human T-cell leukemia virus type 1-like particles. Viruses 8:1-11

Maranga L, Brazao TF, Carrondo MJT (2003) Virus-like particle production at low multiplicities of infection with the baculovirus insect cell system. Biotechnol Bioeng 84:245-253

Martin JL, Cao S, Maldonado JO, Zhang W, Mansky LM (2016) Distinct particle morphologies revealed through comparative parallel analyses of retrovirus-like particles. J Virol 90:8074-8084

Mohsen MO, Zha L, Cabral-Miranda G, Bachmann MF (2017) Major findings and recent advances in virus-like particle (VLP)-based vaccines. Semin Immunol 34:123-132

Monteiro F, Bernal V, Chaillet M, Berger I, Alves PM (2016) Targeted supplementation design for improved production and quality of enveloped viral particles in insect cell-baculovirus expression system. J Biotechnol 233:34-41

Nika L, Wallner J, Palmberger D, Koczka K, Vorauer-Uhl K, Grabherr R (2017) Expression of full-length HER2 protein in Sf9 insect cells and its presentation on the surface of budded virus-like particles. Protein Expr Purif 136:27-38

Nika L, Cuadrado-Castano S, Arunkumar GA, Grünwald-Gruber C, McMahon M, Koczka K, García-Sastre A, Krammer F, Grabherr R (2019) An HER2-displaying virus-like particle vaccine protects from challenge with mammary carcinoma cells in a mouse model. Vaccines 7:1-19

Pastor AR, González-Domínguez G, Díaz-Salinas MA, Ramírez OT, Palomares LA (2019) Defining the multiplicity and time of infection for the production of Zaire Ebola virus-like particles in the insect cell-baculovirus expression system. Vaccine 37:6962-6969

Pereira Aguilar P, González-Domínguez I, Schneider TA, Gòdia F, Cervera L, Jungbauer A (2019) At-line multi-angle light scattering detector for faster process development in enveloped virus-like particle purification. J Sep Sci 42:2640-2649
Pillay S, Meyers A, Williamson AL, Rybicki EP (2009) Optimization of chimeric HIV-1 virus-like particle production in a baculovirus-insect cell expression system. Biotechnol Prog 25:1153-1160

Pinzi S, Leiva D, Arzamendi G, Gandia LM, Dorado MP (2011) Multiple response optimization of vegetable oils fatty acid composition to improve biodiesel physical properties. Bioresour Technol 102: 7280-7288

Puente-Massaguer E, Lecina M, Gòdia F (2018) Nanoscale characterization coupled to multi-parametric optimization of $\mathrm{Hi} 5$ cell transient gene expression. Appl Microbiol Biotechnol 102:10495-10510

Puente-Massaguer E, Badiella L, Gutiérrez-Granados S, Cervera L, Gòdia F (2019) A statistical approach to improve compound screening in cell culture media. Eng Life Sci 19:1-13

Puente-Massaguer E, Lecina M, Gòdia F (2020) Application of advanced quantification techniques in nanoparticle-based vaccine development with the Sf9 cell baculovirus expression system. Vaccine. https://doi.org/10.1016/j.vaccine.2019.11.087

Pushko P, Tretyakova I, Hidajat R, Zsak A, Chrzastek K, Tumpey TM, Kapczynski DR (2017) Virus-like particles displaying H5, H7, H9 hemagglutinins and N1 neuraminidase elicit protective immunity to heterologous avian influenza viruses in chickens. Virology 501:176-182

Ratajczak J, Wysoczynski M, Hayek F, Janowska-Wieczorek A, Ratajczak MZ (2006) Membrane-derived microvesicles: important and underappreciated mediators of cell-to-cell communication. Leukemia 20:1487-1495

Sander L, Harrysson A (2007) Using cell size kinetics to determine optimal harvest time for Spodoptera frugiperda and Trichoplusia ni BTI-TN5B1-4 cells infected with a baculovirus expression vector system expressing enhanced green fluorescent protein. Cytotechnology 54:35-48

Senger T, Schädlich L, Gissmann L, Müller M (2009) Enhanced papillomavirus-like particle production in insect cells. Virology 388:344-353

Shoja Z, Tagliamonte M, Jalilvand S, Mokhtari-Azad T, Hamkar R, Shahmahmoodi S, Rezaei F, Tornesello M, Buonaguro FM, Buonaguro L, Nategh R (2013) Development of a stable insect cell line constitutively expressing rotavirus VP2. Virus Res 172:66-74

Tagliamonte M, Visciano ML, Tornesello ML, De Stradis A, Buonaguro FM, Buonaguro L (2010) Constitutive expression of HIV-VLPs in stably transfected insect cell line for efficient delivery system. Vaccine 28:6417-6424

Thompson CM, Petiot E, Mullick A, Aucoin MG, Henry O, Kamen AA (2015) Critical assessment of influenza VLP production in Sf9 and HEK293 expression systems. BMC Biotechnol 15:1-12

Venereo-Sanchez A, Gilbert R, Simoneau M, Caron A, Chahal P, Chen W, Ansorge S, Li X, Henry O, Kamen A (2016) Hemagglutinin and neuraminidase containing virus-like particles produced in HEK-293 suspension culture: an effective influenza vaccine candidate. Vaccine 34:3371-3380

Vidigal J, Fernandes B, Dias MM, Patrone M, Roldão A, Carrondo MJT, Alves PM, Teixeira AP (2018) RMCE-based insect cell platform to produce membrane proteins captured on HIV-1 Gag virus-like particles. Appl Microbiol Biotechnol 102:655-666

Visciano ML, Diomede L, Tagliamonte M, Tornesello ML, Asti V, Bomsel M, Buonaguro FM, Lopalco L, Buonaguro L (2011) Generation of HIV-1 virus-like particles expressing different HIV1 glycoproteins. Vaccine 29:4903-4912 5

Wilde M, Klausberger M, Palmberger D, Ernst W, Grabherr R (2014) Tnao38, High Five and Sf9-evaluation of host-virus interactions in three different insect cell lines: baculovirus production and recombinant protein expression. Biotechnol Lett 36:743-749

Young KR, Ross TM (2006) Elicitation of immunity to HIV type 1 Gag is determined by Gag structure. AIDS Res Hum Retrovir 22:99-108

Publisher's note Springer Nature remains neutral with regard to jurisdictional claims in published maps and institutional affiliations. 